\title{
Effect of Fascial Manipulation on Glenohumeral Internal Rotation Deficit in Overhead Athletes - A Randomized Controlled Trial
}

\author{
Nelson P Mathew, Prabu Raja G, F. Davis \\ Department of Exercise and Sports Sciences, Manipal College of Health Professions, Manipal Academy \\ of Higher Education
}

\author{
CORRESPONDING AUTHOR: \\ Prabu Raja G \\ Department of Exercise and \\ Sports Sciences \\ Manipal College of Health Professions \\ Manipal Academy of Higher Education \\ Karnataka, India 576104 \\ Phone: +91 8867591333 \\ E-mail: prabu.raja@manipal.edu \\ DOI: \\ 10.32098/mltj.01.2020.03
}

LEVEL OF EVIDENCE: 1B

\begin{abstract}
SUMMARY
Background. Glenohumeral Internal Rotation Deficit (GIRD) is common among overhead athletes and is considered as one of the risk factors for upper extremity injuries. GIRD is usually managed by stretching and soft tissue release of the posterior shoulder capsule. Fascial manipulation is a manual therapy technique used in the management of musculoskeletal disorders. This study compared the efficacy of fascial manipulation (FM) with posterior capsular ball release and stretching on GIRD.

Purpose. The purpose of this study is to determine the effect of Fascial Manipulation (FM) on the Internal Rotation Range of Motion (IRROM) in athletes with GIRD. Since studies have shown a possible association between GIRD and shoulder injuries, improving the IRROM in athletes with GIRD may help in reducing the risk of shoulder injuries. Thus, FM may be used as an effective strategy in increasing IRROM in overhead athletes with GIRD, thus preventing upper extremity injuries.

Study Design. Randomized controlled trial.

Methods. Asymptomatic overhead athletes with GIRD more than $20^{\circ}$ when compared with the non-dominant shoulder were randomly assigned to two groups. The experimental group has received three sessions of FM treatment in two weeks. FM applied to densified Centre of Coordination (CC) points located on the myofascial sequences for 5 to 8 minutes at each CC point. The control group has received three sessions of posterior shoulder capsule release using a tennis ball under supervision. Along with the ball release, the therapist taught home-based, unsupervised sleeper, and cross-body adduction stretches, for the control group. A universal goniometer was used to measure the IRROM before and after all three treatment sessions in both groups.

Results. There were no statistically significant differences between the control and experimental groups $(p<0.05)$. However, immediate improvement in the IRROM following FM was more substantial in the experimental group following each session.

Conclusions. This study indicates that FM may be used as an adjunct to stretching in asymptomatic participants with GIRD to increase the IRROM. There is a scope for future studies to be done to investigate the effect of FM on symptomatic overhead athletes with GIRD.
\end{abstract}

\section{KEY WORDS}

Fascial manipulation (FM); Glenohumeral internal rotation deficit (GIRD); internal rotation range of motion

\section{INTRODUCTION}

Overhead throwing athletes (OTA) tend to have restricted Internal rotation Range of Motion (IRROM) leading to Glenohumeral Internal Rotation Deficit (GIRD). Anatomical GIRD results in the loss of IRROM, along with an equivalent increase in ERROM due to increased humeral retro- version (HR) $(1,2,3,6)$. The increased $H R$ allows higher shoulder External Rotation Range of Motion (ERROM) during the cocking phase, thereby assisting the shoulder in reaching high rotational velocities. Along with the bony adaptations that occur in OTA, few studies have demonstrated soft tissue adaptations where there is an increase in 
the stiffness of the shoulder posterior capsule and the rotator cuff muscles. The substantial increase in the thickness of the posterior capsule may occur due to repetitive strain injuries that occur over a period of repeated overhead throwing movements. This leads to the formation of reactive scar tissue over the posterior capsule and shoulder musculature thereby resulting in $\operatorname{GIRD}(3,4,5)$.

Pathologic GIRD occurs when there is a deficit in the Total Arc of Rotational Motion (TARM) where the IRROM loss is more than the ERROM gain. The deficit in the TARM results in a higher risk of injury in the shoulder and elbow of overhead athletes $(1,6)$. In addition, an imbalance in the strength of internal and external rotators of the shoulder may alter the throwing mechanics and compromise scapulothoracic and glenohumeral joint stability. This muscular imbalance is also considered as one of the risk factors of injury in throwers (7).

Khalil et al, have indicated that there were no differences in the IRROM between dominant and non-dominant arm during pre-season evaluation, whereas subsequent postseason measurements have shown significant reduction in the IRROM of the dominant arm when compared with non-dominant arm. Even though anatomic changes in the ulnar collateral ligaments of the elbow have shown some potential to resolve during off-season, GIRD which had developed during the competitive season, were found to progress and did not resolve even after off-season rest. Since there is a plausible association between the UCL adaptations and GIRD and the progressive adaptations of GIRD in OTA, it is vital to improve GIRD to reduce the risk of shoulder and elbow injuries in OTA (8).

Joint mobilization, shoulder capsular release using balls, and stretching of the posterior capsule are the conventional treatment strategies used in the management for GIRD (3, 6). These traditional treatment methods focus only on the rigid posterior capsular structure. Although muscle-energy technique and myofascial release are used in the treatment of GIRD, further research is required to study their effectiveness (3). Nevertheless, Bailey et al have shown that an instrument-assisted soft tissue mobilization along with stretching have shown a significant improvement in the IRROM and TARM. Along with this, there was a substantial reduction in the stiffness of the posterior rotator cuff muscles thus showing an association between the rotator cuff stiffness and GIRD. Thus, reducing the stiffness by using manual therapy techniques on soft tissues may improve GIRD that are associated with risk of injuries (9).

The pathological changes in the deep fascia are considered to be a poteno study has investigated the biomechanical consequences of glenohumeral internal rotation deficit (GIRDntial source of musculoskeletal pain and dysfunctions (10). Fascial Manipulation (FM) works on the entire myofascial sequences of the upper quarter (UQ). Based on the principle of FM, the UQ is divided into various segments, which include head, neck, thorax, scapula, humerus, elbow, carpus, and digits. Each segment consists of six myofascial units (MFU). MFU consists of muscle fibers, the associated deep fascia, and the articulation that they move on each plane (11). These MFU of the different segments are linked to form myofascial sequences (MFS), thus creating a myofascial continuum. The MFS is considered to monitor the movement of several segments in one plane. Within each MFU, the centre of coordination (CC) is the point of convergence of the vectorial, muscular forces that act on a body segment. CC points of the reciprocal antagonistic MFU were palpated for densification on the myofascial sequences $(10,11,12)$. The adaptive changes of these myofascial continuum (MC) may occur due to repeated overhead activity rather than the posterior capsular structures alone. The FM restores the gliding of collagen and elastin fibers within the ground substance by the use of heat generated from the manipulation at CC points. These adaptations may propagate along an MFS, re-establishing the balance $(11,12)$. Hence, we aimed to compare the efficacy of FM, which focusses on MC of UQ, with the usual care (posterior capsular ball release and sleeper stretch) that focuses only on the posterior capsule on IRROM in overhead athletes with GIRD.

\section{MATERIALS AND METHODS}

\section{Trial design}

This study was designed as a randomized, active-controlled, outcome assessor-blinded, parallel-group, two-arm trial with an allocation ratio of 1:1.

\section{Participants}

University level overhead recreational athletes. Forty-three asymptomatic overhead athletes with shoulder internal rotation deficit $\geq 20^{\circ}$ when comparing to the non-dominant side, between 18 to 45 years of age, were included in the study. The demographic data of all participants were collected and documented. Athletes with symptoms of neck pain or shoulder pain and athletes with skin lesions were excluded from the study.

\section{Procedure}

This study obtained approval from the Institutional Ethics Committee (IEC 331/2016). All authors who were involved 
in this study have followed the international ethical principles as well as the ethical standards of the Muscle, Ligaments and Tendons Journal (13).

University level recreational athletes were screened. The written informed consent was taken after identifying asymptomatic individuals with GIRD. Subjects were randomly assigned to experimental and control groups. The flow of participants is shown in figure $\mathbf{1}$.

\section{Interventions}

Fascial manipulation. After the identification of the densified Centre of Co-ordination (CC) points along the Intra and Extra myofascial sequences, the therapist treated the clients with FM using knuckle or pad of the fingers or using olecranon process of the elbow as shown in figure 2. Fascial manipulation was done at each densified CC points for about 5-8 minutes $(10,11,16)$. On average, at least four densified points on each myofascial sequence were identified. Thus, a total of 8-10 points were manipulated on each athlete, including both the antagonistic intra and extra myofascial sequences. The total duration of FM lasted for about 45 minutes to an hour during each session.

Shoulder posterior capsular ball release and Stretching. A self-release was done using a tennis ball on the posterior shoulder capsular region for about 90 seconds for three sessions under the supervision of the therapist (figure 3). An ischemic compression was done on the posterior shoulder capsular region using a tennis ball without any movement, which results in temporary obstruction of local blood flow followed by the influx of oxygenated blood as soon as the pressure is released. This compression could normalize the biomechanical properties of muscle fibers, thus decreasing the injury risk (17).

Sleeper stretch was done in a side-lying position with the shoulder in 90-degree abduction, elbow at 90-degree flex-

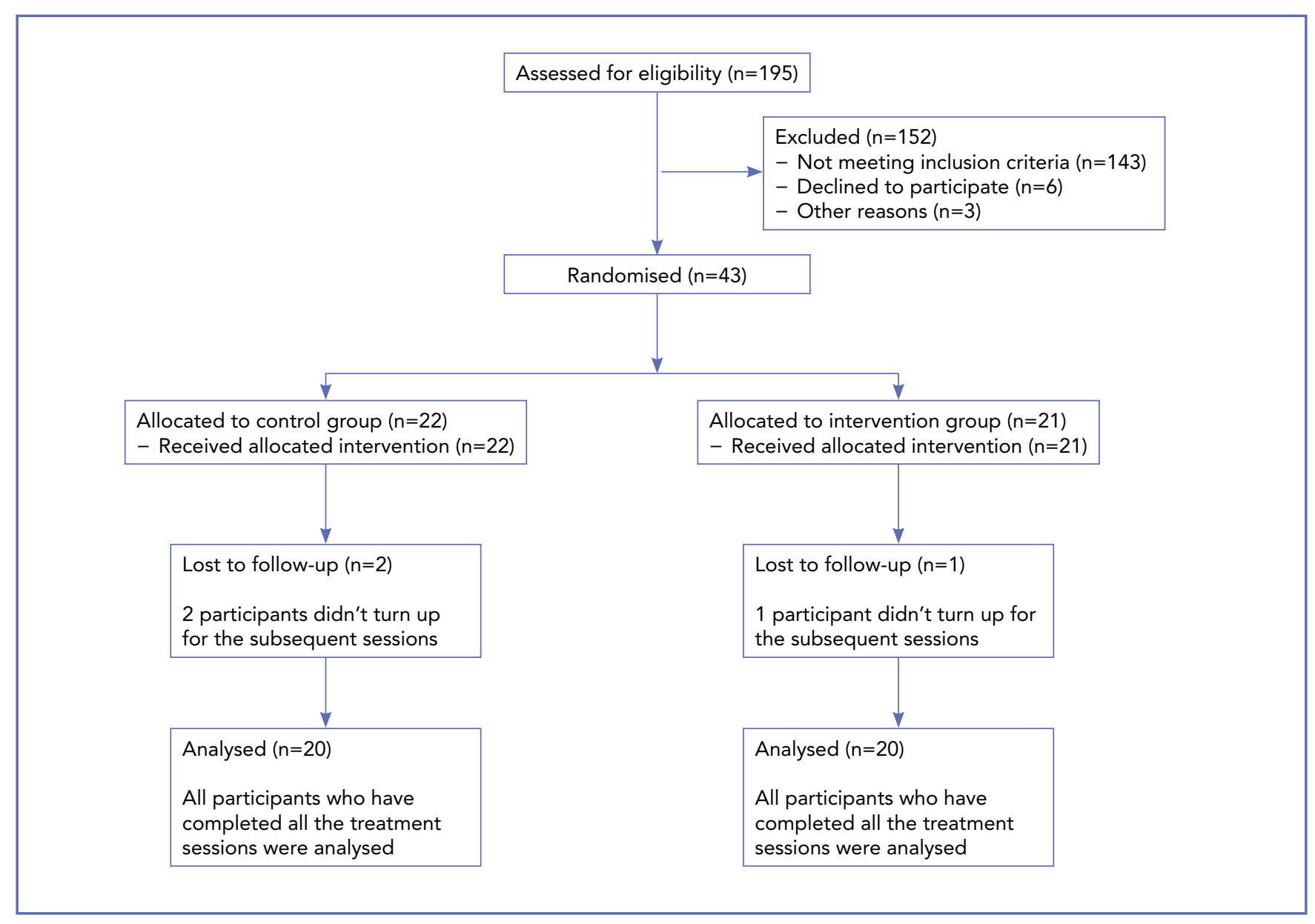

Figure 1. CONSORT Flow Diagram. 


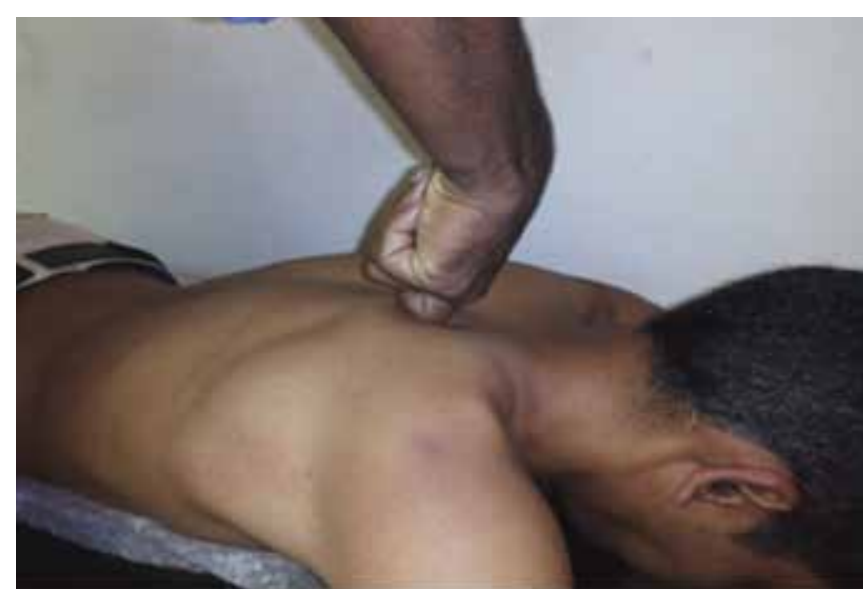

Figure 2. Fascial manipulation.

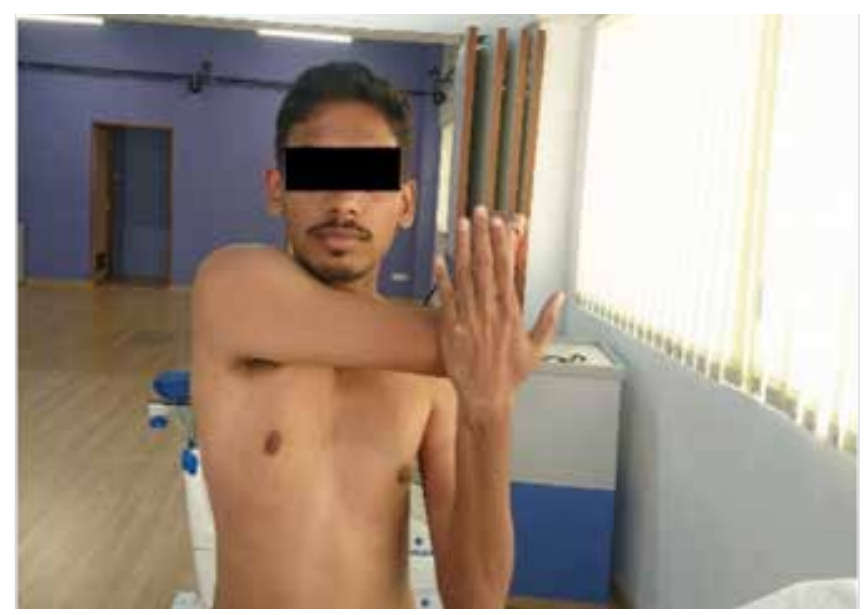

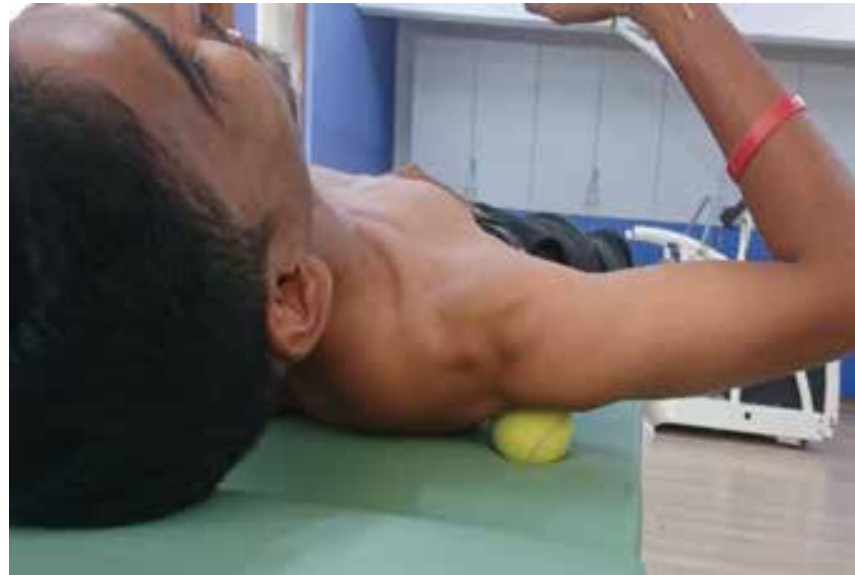

Figure 3. Posterior shoulder capsular release.

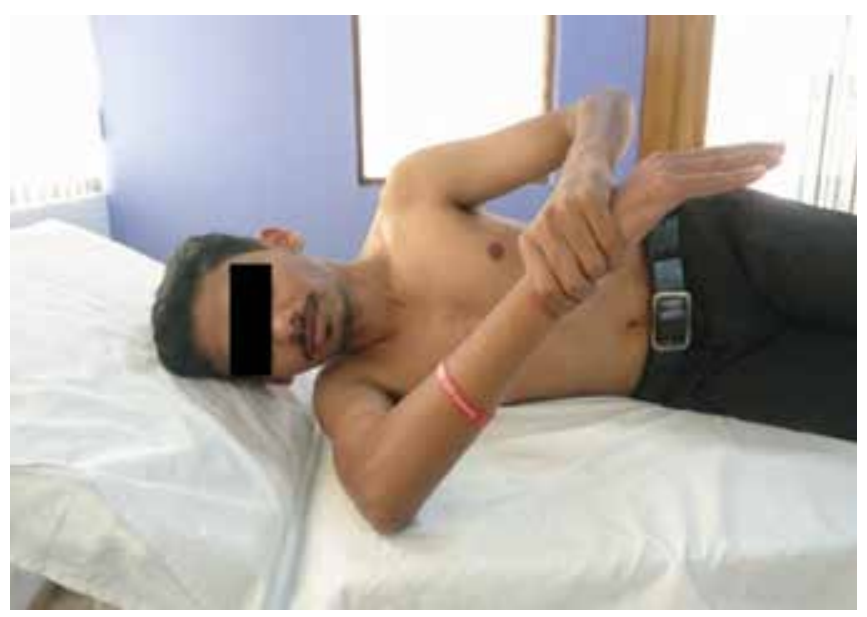

Figure 4. Stretching (Cross body adduction and sleeper stretches).

ion, and then performing shoulder internal rotation. Crossbody adduction stretch was done in sitting position by taking the arm into horizontal adduction. Three sets of the each stretch positions were held for 30 seconds with a 1-minute break between the sets as shown in figure 4 (15, 17, 19).

\section{Outcome measures}

IRROM measurements. The internal rotation ROM was measured by having the participant on the affected side with the shoulder and elbow kept at 90 degrees of flexion. The examiner placed the scapula at a position of maximal adduction or retraction by reaching around the participant and pulling the lateral scapular border towards the spine, and the ROM was measured using the goniometer $(1,18)$.

\section{Data analysis}

The recorded data were analyzed using SPSS version 16. Descriptive statistics were used to measure baseline demographic data. Repeated measures ANOVA was used to calculate the differences in $\mathrm{ROM}$ with the level of significance $\mathrm{p}$ $\leq 0.05$. Kolmogorov-Smirnov test for normality showed that data followed the normal distribution. Hence, a parametric test was used to analyze the outcome variable. An independent $\mathrm{t}$-test was used to find the difference between the groups, and repeated measure ANOVA was done to identify the differences within the group.

\section{RESULTS}

Participant's baseline characteristics are shown in table I. Repeated measures ANOVA was used to analyze the data, 
Table I. Demographic characteristics of the study participants $(n=20)$.

\begin{tabular}{lcc}
\hline Variables & $\begin{array}{c}\text { Experimental Group } \\
\text { Mean } \pm \text { SD }\end{array}$ & $\begin{array}{c}\text { Control Group } \\
\text { Mean } \pm \text { SD }\end{array}$ \\
\hline Age in years & $23 \pm 2.89$ & $22 \pm 3.05$ \\
\hline Height in cms* & $171 \pm 4.9$ & $170 \pm 4.06$ \\
\hline Weight in $\mathrm{kgs}^{\ddagger}$ & $68 \pm 3$ & $66 \pm 5.72$ \\
\hline
\end{tabular}

*cms - Centimeters

$\ddagger$ kgs- Kilograms

and there is a significant improvement in the IRROM between the treatment sessions in both the groups. Figure 5 shows that there is a marked improvement in the ROM between sessions one and two $(\mathrm{p} \leq 0.05)$ and between sessions one and three $(\mathrm{p} \leq 0.05)$ as well as between the second and third sessions $(\mathrm{p} \geq 0.05)$. However, there were no statistically significant differences between the control and experimental group $(\mathrm{p} \geq 0.05)$. The graph indicates that there is an increase in IRROM seen in both groups. There was an immediate improvement in IRROM following each session in the experimental group. Still, the improvement in IRROM did not last long as there was a decrease in the IRROM during the follow-up sessions. In the control group, even though there were no significant immediate effects, there was a gradual improvement in the IRROM.

\section{DISCUSSION}

Although, this study showed a substantial improvement of IRROM in athletes with GIRD in both the experimental and control groups, there is no statistically significant difference between the groups after the end of three sessions. However, the immediate improvement in the IRROM following FM is more significant as compared to the control group, which received stretching and ball release. During the follow-up sessions, the IRROM decreased during the pre-treatment measurements and increased immediately following the treatment sessions. The considerable short-term improvement in the IRROM, reduces during the follow-up evaluation before the subsequent treatment sessions in the intervention group. Although there is a clinically significant improvement in the IRROM within the experimental group, the effect did not last long as there were reductions in IRROM during follow-ups. Thus, FM might be used as an effective strategy in the immediate improvement of IRROM on GIRD. In order to maintain the IRROM gain following FM, it is important to add home-based stretching protocol along with FM. As GIRD is considered as one of the risk factors of shoulder injuries in overhead athletes, improving the GIRD may help in the prevention of shoulder injuries in overhead athletes.

Since alterations of the deep muscular fascia and their myofascial continuum may result in musculoskeletal dysfunctions, FM provides a rationale for the treatment of specific areas of deep muscular fascia called CC points along the myofascial continuum (10). Thus, FM on the myofascial continuum of the upper quadrant may lead to increased IRROM in our study participants.

A systematic review of the effect of stretching on GIRD showed substantial improvement in GIRD when stretching was done for more than three weeks. Although this review focused on injury prevention for asymptomatic overhead

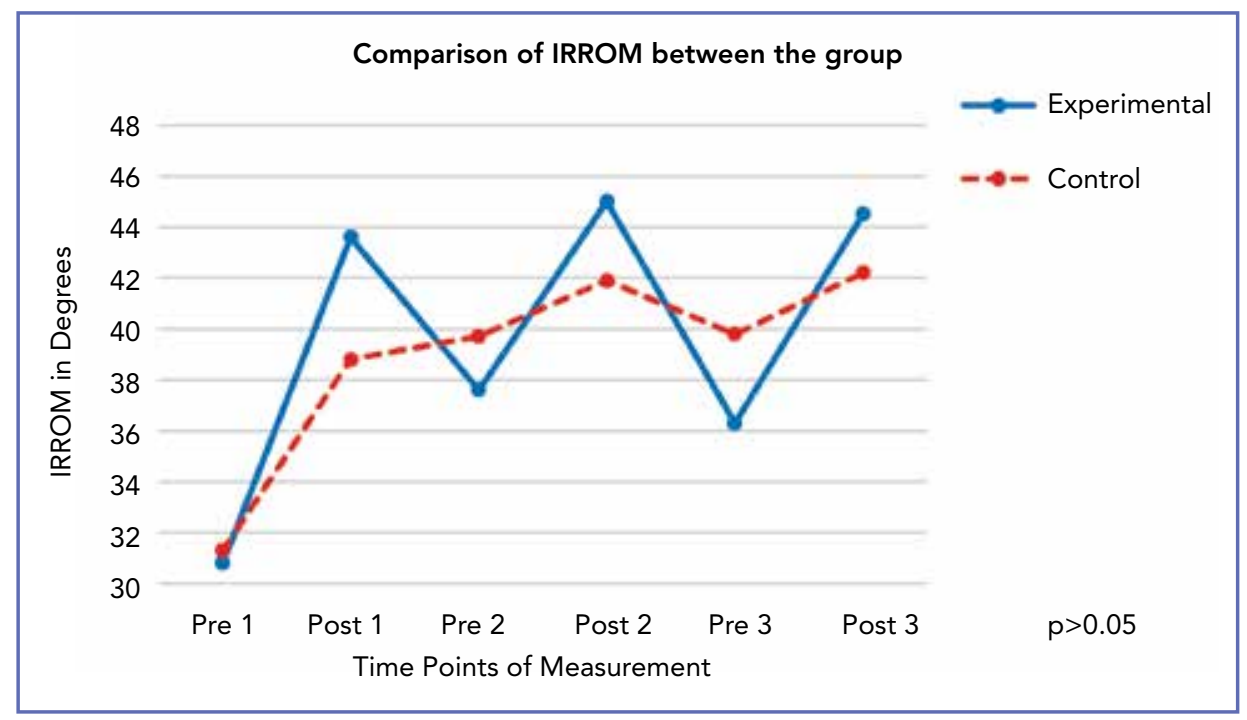

Muscles, Ligaments and Tendons Journal 2020;10 (1)
Figure 5. Between group comparison of IRROM. Pre 1- IRROM measurement prior to 1st treatment session; Post 1- IRROM measurement immediately following 1st treatment session; Pre 2 IRROM measurement prior to 2 nd treatment session; Post 2 - IRROM measurement immediately following 2nd treatment session; Pre 2 IRROM measurement prior to $3 \mathrm{rd}$ treatment session; Post 2 - IRROM measurement immediately following 3rd treatment session. 
athletes with GIRD, future studies should focus on the effect of improvement in the GIRD and their influence on the injury rates $(15,17)$.

Our study showed there was a gradual improvement in the IRROM throughout all the sessions in the conventional treatment group (Ball release/ Stretching). The progressive increment in the IRROM could be due to the addition of a home-based stretching program, but the immediate effects were not as significant as the experimental group.

The FM group in this study showed an improvement in the IRROM immediately following the treatment session. This improvement in IRROM could be attributed to the decrease in the viscosity of Hyaluronic Acid (HA) occurring in the deep fascia. In the experimental group, FM applied over the CC points in the Intra and Extra myofascial sequences, increases the local temperature in the deep fascia and its continuum. The local rise in temperature due to kneading, improve the pliability of tissues in these myofascial sequences, which might have enhanced the flexibility of these connective tissues and thus improving the IRROM $(10,11,16)$.

Even though GIRD is attributed the skeletal adaptation such as increased humeral retroversion, most of the adaptive changes may also occur due to the formation of adhe-

\section{REFERENCES}

1. Cieminski CJ., Klaers H., Kelly SM., Stelzmiller MR., Nawrocki TJ., Indrelie AJ. Total arc of motion in the side-lying position: Evidence for a new method to assess glenohumeral internal rotation deficit in overhead athletes. International Journal of Sports Physical Therapy. 2015; 10(3), 319-31.

2. Gates JJ., Gupta A., McGarry MH., Tibone JE., Lee T. Q. The effect of glenohumeral internal rotation deficit due to posterior capsular contracture on the passive glenohumeral joint motion. American Journal of Sports Medicine. 2012; 40(12), 2794-2800.

3. Rose MB., Noonan T. Glenohumeral internal rotation deficit in throwing athletes: current perspectives. J Sports Med. 2018; 19(9), 69-78.

4. Hellem A., Shirley M., Schilaty N., Dahm D. Review of shoulder Range of Motion in the throwing athlete: distinguishing normal adaptations from pathologic deficits. Current Reviews in Musculoskeletal Medicine 2019; 12: 346-355.

5. Wilk KE., Macrina LC., Fleisig GS., Porterfield R., Simpson CD., Harker P., et al. Correlation of glenohumeral internal rotation deficit and total rotational motion to shoulder injuries in professional baseball pitchers. Am J Sports Med. 2011;39(2):329-35.

6. Tokish JM., Curtin MS., Kim YK., Hawkins RJ., Torry MR. Glenohumeral internal rotation deficit in the asymptomatic professional pitcher and its relationship to humeral retroversion. Journal of Sports Science and Medicine. 2008; 7(1), 78-83. sions in the soft tissues around the shoulder and scapular region. Thus soft tissue therapy techniques become an integral part of athletic injury prevention and rehabilitation.

Considering the immediate improvement of IRROM with FM and the gradual and consistent increase of IRROM with the stretching, FM may be useful in subjects with GIRD. Nevertheless, the efficacy of FM in symptomatic overhead throwing athletes with GIRD remains unanswered which necessitates future research.

\section{CONCLUSIONS}

As there is a possible association between GIRD and shoulder injuries, improving the GIRD may help in reducing the risk of shoulder injuries. Thus, FM can be used along with stretching of shoulder posterior capsule in the management of GIRD, thereby preventing shoulder injuries in overhead athletes. Adding a posterior capsular stretching would augment the immediate gain in IRROM with FM, which may reduce the risk of shoulder injuries.

\section{CONFLICT OF INTERESTS}

The authors declare that they have no conflict of interests.

7. Pontaga I. Shoulder external/internal rotation peak torques ratio side-asymmetry, mean work and power ratios balance worsening due to different fatigue resistance of the rotator muscles in male handball players. MLTJ. 2018: 8(4): 513-19.

8. Khalil LS., Okoroha KR., Jildeh TR., Matar RN., Fidai MS., Tramer JS., Ansok C., Scher C., Van Holsbeeck M., Makhni EC., Moutzouros V. Journal of Shoulder and Elbow surgeryOpen Access. 2019: 15; 3(4):338-343.

9. Bailey LB., Shanley E., Hawkins R., Beattie PF., Fritz S. Kwartowitz D., Thigpen CA. Mechanisms of Shoulder Range of Motion Deficits in Asymptomatic Baseball Players. Am J Sports Med. 2015;43 (11): 2783-93.

10. Day JA., Stecco C., Stecco A. Application of Fascial Manipulation technique in chronic shoulder pain-Anatomical basis and clinical implications. Journal of Bodywork and Movement Therapies. 2009; 13, 128-135.

11. Stecco L. Fascial manipulation for musculoskeletal pain. Piccin. 2004; Padova.

12. Schleip R., Findley TW., Chaitow L., Huijing P. Fascia - The Tensional Network of the Human Body. The Science and clinical applications in manual and movement therapies. Churchill Livinstone. 2009; Elsevier.

13. Padulo J., Oliva F., Frizziero A., Maffulli N. Muscles, Ligaments and Tendons Journal - Basic principles and recommendations in clinical and field Science Research: 2018 update. MLTJ. 2018; 8(3): $305-307$. 
14. Manske R., Wilk KE., Davies G., Ellenbecker T., Reinold M. Glenohumeral motion deficits: Friend or Foe? Int J Sports Phys Therapy. 2013;8(5), 537-53.

15. Nichols J., Calver S., Chester R. Are stretches effective in the prevention and treatment of glenohumeral internal rotation deficit? Physical Therapy Reviews. 2012; 17, 261-70.

16. Ercole B., Stecco A., Day JA., Stecco C. How much time is required to modify a fascial fibrosis? Journal of Bodywork and Movement Therapies. 2010; 14(4), 318- 25.

17. Manske RC., Meschke M., Porter A., Smith B., Reiman M. A randomized controlled single-blinded comparison of stretch- ing versus stretching and joint mobilization for posterior shoulder tightness measured by internal rotation motion loss. Sports Health. 2010; 2(2), 94-100.

18. Torres RR., Gomes JL. Measurement of glenohumeral internal rotation in asymptomatic tennis players and swimmers. American Journal of Sports Medicine. 2009; 37(5), 1017-23.

19. Cools, A.M., Johansson, F.R., Borms, D., Maenhout, A., 2015. Prevention of shoulder injuries in overhead athletes: a sciencebased approach. Brazilian Journal of Physical Therapy. (5), 331-9. 